\title{
POSITIVE SOLUTIONS OF SEMILINEAR EQUATIONS IN CONES
}

\author{
HENRIK EGNELL
}

\begin{abstract}
In this paper we consider the problem of finding a positive solution of the equation $\Delta u+|x|^{\nu} u^{(n+2+2 \nu) /(n-2)}=0$ in a cone $\mathscr{C}$, with zero boundary data. We are only interested in solutions that are regular at infinity (i.e. such that $u(x)=o\left(|x|^{2-n}\right)$, as $\left.\mathscr{C} \ni x \rightarrow \infty\right)$. We will always assume that $\nu>-2$.

We show that the existence of a solution depends on the sign of $\nu$ and also on the shape of the cone $\mathscr{C}$.
\end{abstract}

\section{INTRODUCTION}

Let $\Omega$ be a connected domain on the unit sphere $\Sigma^{n-1}$ in $\mathbf{R}^{n}$, and define the open cone $\mathscr{C}=\mathscr{C}_{\Omega}=\left\{x \in \mathbf{R}^{n}: x=r \theta, \theta \in \Omega\right.$ and $\left.r>0\right\}$. We will assume that $\overline{\mathscr{C}} \neq \mathbf{R}^{n}$.

In the paper [BE] (see also [BaL]) Bandle and Essén studied positive solutions of the equation

$$
\Delta u+|x|^{\nu} u^{p}=0 \text { in } \mathscr{C}, \quad u=0 \text { on } \partial \mathscr{C} \backslash\{0\} .
$$

Here $\nu \in \mathbf{R}$ and $p>1$.

They defined a solution to be regular at 0 if $u(x) \rightarrow 0$, as $\mathscr{C} \ni x \rightarrow 0$. In view of the invariance under the Kelvin transform, they defined a function to be regular at infinity if $\left.u(x)|| x\right|^{n-2} \rightarrow 0$, as $\mathscr{C} \ni x \rightarrow \infty$. Finally a solution is called regular if it is regular at both infinity and zero.

In Theorem 4.1 they proved that equation $(0.1)$ has no solutions such that $|u(x)||x|^{(\nu+2) /(p-1)} \rightarrow 0$, as $C \ni x \rightarrow 0$ and as $\mathscr{C} \ni x \rightarrow \infty$. Especially if $p+1 \neq 2(n+\nu) /(n-2)$, and $\nu \geq-2$ then $(0.1)$ cannot have a regular solution.

However, the situation when $p+1=2(n+\nu) /(n-2)$ remained open. In this paper we shall see that the conclusion will depend on the sign of $\nu$ and if $\nu$ is nonnegative also on the shape of the cone $\mathscr{C}_{\Omega}$ (i.e. the shape of $\Omega$ ). Thus in this paper we will study the special case of finding a positive solution of

$$
\begin{aligned}
& \Delta u+|x|^{\nu} u^{(n+2+2 \nu) /(n-2)}=0 \text { in } \mathscr{C}, \quad \text { with } \nu>-2, \\
& u=0 \text { on } \partial \mathscr{C}, \text { and } u(x)=o\left(|x|^{2-n}\right), \quad \text { as } \mathscr{C} \ni x \rightarrow \infty .
\end{aligned}
$$

From now on the exponent $p$ will always be chosen so that $p+1=$ $2(n+\nu) /(n-2)$. If $\nu \leq 0$, then this is the only exponent $p$ for which $\mathscr{D}_{0}^{1,2}(\mathscr{C}) \hookrightarrow L^{p+1}\left(|x|^{\nu} d x\right)$, is continuous. If $\nu$ is positive the same is true

Received by the editors December 11, 1989.

1980 Mathematics Subject Classification (1985 Revision). Primary 35J20, $35 \mathrm{~J} 25$.

Supported in part by NSF Grant DMS-8914778. 
if we restrict to radial functions and replace $\mathscr{C}$ with $\mathbf{R}^{n}$. Here $\mathscr{D}_{0}^{1,2}(\mathscr{C})$ is defined as the completion of $C_{0}^{\infty}(\mathscr{C})$ in the norm $\|\nabla u\|_{2}$.

If $\nu \leq-2$, then the exponent $p \leq 1$ and the problem is different. In fact if $\nu=-2$, then there are no regular solutions. This is proved at the end of $\S 1$. On the other hand if $-2<\nu<0$, then there is always a solution of $(0.2)$ (Theorem 2 below).

To prove existence we will use variational methods. The variational approach gives us a positive solution of the following weak form of $(0.2)$.

$\left(0.2^{\prime}\right) \quad$ Find $u \in \mathscr{D}_{0}^{1,2}(\mathscr{C})$ satisfying $\Delta u+|x|^{\nu} u^{p}=0$ in the weak sense.

If $\nu \leq 0$, then regularity theory shows that a solution of $\left(0.2^{\prime}\right)$ is a classical solution of $(0.2)$. For the regularity at thi crigin, we can first prove that the solution is bounded using Moser iterations with weighted Sobolev inequalities as in [EG2]. Then since $|x|^{\nu} \in L_{\mathrm{loc}}^{p}$ for some $p>n / 2$ near the origin it follows that $u$ is Hölder continuous at the origin [GT, Chapter 8]. The regularity at infinity follows from the fact that $\left(0.2^{\prime}\right)$ is invariant under the Kelvin Transform (see the end of $\S 2$ in the present paper or [BE]).

For a more precise description of the behaviour of regular solutions near the origin and near infinity, see Theorem 3.3 in [BE].

In the case when $\nu$ is positive we have to restrict the class of functions in the variational problem. The weak solution we obtain in this class turns out to be a classical solution of $(0.2)$ too.

Our first result extends the nonexistence result in $[\mathrm{BE}]$ mentioned above to a certain class of cones, provided $\nu$ is nonnegative.

Theorem 1. Assume that $\nu \geq 0$. If the cone $\mathscr{C}$ is contained in a half space with normal $x_{0} \in \mathscr{C}$ and if $\mathscr{C}$ is starshaped with respect to $x_{0}$. Then there are no solutions of equation (0.2). In the special case when $\nu=0$, the conclusion holds without the assumption that $\mathscr{C}$ is contained in a half space.

Remark. As pointed out by M. Essen, under the conditions on $\mathscr{C}$ stated in the theorem, there are no solutions of $(0.1)$ such that $u(x)|x|^{(\nu+2) /(p-1)} \rightarrow 0$ as $\mathscr{C} \ni x \rightarrow 0$ and as $\mathscr{C} \ni x \rightarrow \infty$.

The proof of Proposition 1 is based on a Pohozaev type identity, which was also used in [BE].

The result above excludes the case when $\nu \in(-2,0)$. In this case we have the following positive result.

Theorem 2. If $-2<\nu<0$, then equation ( 0.2$)$ has a solution. This solution can be obtained as a minimizer of the quotient $\|\nabla u\|_{2}^{2} /\|u\|_{p+1,|x|^{\nu}}^{2}$ over $\mathscr{D}_{0}^{1,2}(\mathscr{C})$.

Corollary of Theorem 2. Let $-2<\nu<0$, and consider the inequality

$$
S\|u\|_{p+1,|x|^{\nu}}^{2} \leq\|\nabla u\|_{2}^{2} \text { for } u \in \mathscr{D}_{0}^{1,2}(\mathscr{C}),
$$

with best constant $S$. Then there is a nontrivial function $u \in \mathscr{D}_{0}^{1,2}(\mathscr{C})$ that gives equality.

We remark that in the endpoint cases the inequality is strict, unless $u \equiv 0$. If $\nu=-2$, the inequality is called Hardy's inequality, and at the end of $\S 1$ we prove that equality cannot be attained. If $\nu=0$ and $\mathbf{R}^{n} \backslash \mathscr{C}$ has positive capacity, then it is well known that we cannot have equality. The reason is 
that the best constant $S$ is independent of the domain $C$. Furthermore, if we take $\mathscr{C}=\mathbf{R}^{n}$ then the only function giving equality is $u(x)=\left(1+|x|^{2}\right)^{(2-n) / 2}$ (modulo translations, dilations and a multiplicative constant).

The proof of Theorem 2 is based on the Concentration-Compactness principle [L1].

In the case when $\nu \geq 0$ we can elaborate a little bit further to see that we need some conditions on $\mathscr{C}$ to conclude that we do not have a solution.

The following example is in the same spirit as that given by Kazdan and Warner $[\mathrm{KW}]$ in a finite domain.

Theorem 3. If $\mathscr{C}=\left\{(y, z) \in \mathbf{R}^{n-1} \times \mathbf{R}: z>0, d z<|y|<D z\right\}$, where $d$ and $D$ are positive constants, then (0.2) has a solution. This solution is invariant under rotation around the $z$-axis.

Let us remark that in the proof of Theorem 3 we still have a problem with lack of compactness, due to the dilation invariance. In the example given by Kazdan and Warner, the compactness was restored by restricting to radial functions.

When $\nu=0$ we can use the arguments from [CO] and [DI] (for results related to these see [DA] or [PA]). However, since we are working in unbounded domains, some modifications of the argument are needed.

Theorem 4. Take $\nu=0$ and assume that $\mathscr{C} \subset \mathbf{R}_{+}^{n}=\left\{(y, z) \in \mathbf{R}^{n-1} \times \mathbf{R}: z>\right.$ $0\}$. Then there is an $\alpha=\alpha(n)>1$ such that if

$$
\mathscr{C}_{\alpha}=\left\{(y, z) \in \mathbf{R}^{n-1} \times \mathbf{R}: \frac{1}{\alpha}|y|<z<\alpha|y|\right\} \subset \mathscr{C},
$$

and

$$
\mathscr{C}_{\alpha}^{\prime}=\left\{(y, z) \in \mathbf{R}^{n-1} \times \mathbf{R}: \alpha|y|<z\right\} \not \subset \overline{\mathscr{C}} .
$$

Then (0.2) has a solution. Put $V_{\delta}=\left\{\left(y_{1}, y^{\prime}, z\right) \in \mathbf{R} \times \mathbf{R}^{n-2} \times \mathbf{R}: \delta y_{1}>\left|y^{\prime}\right|\right\}$, and assume that $n>4$. Then there are numbers $\alpha=\alpha(n)>1$ and $\delta=$ $\delta(\alpha, n)>0$ such that if $\mathscr{C}_{\alpha} \backslash V_{\delta} \subset \mathscr{C}$ and $\mathscr{C}_{\alpha}^{\prime} \not \subset \overline{\mathscr{C}}$. Then (0.2) has a solution.

Note that in the first part in Theorem $4, \Omega=\mathscr{C} \cap \Sigma^{n-1}$ cannot be contractible, while in the second part this is possible. In fact the first part is contained in Theorem 5 below. Of course the second part contains the first part, however we wrote down both statements to compare it with the analogous statements given by Coron and Ding for the problem in a finite domain. Also the proof of the second part is based on that of the first part.

The requirement, $n>4$, in the second part in Theorem 4 is due to some technicalities. Ding considered the equation $\Delta u+u^{(n+2) /(n-2)}=0$, with zero boundary data, in a bounded domain. He constructed a contractible domain where the equation has positive solution, provided the dimension $n>3$. In our case we need one extra dimension since we are working in cones.

Dancer [DA] has constructed a bounded contractible domain, in which there is a solution. His example works in all interesting dimensions (i.e. $n \geq 3$ ) (see also [PA]). It seems to be possible to apply the method used in [DA], to obtain a cone $\mathscr{C}_{\Omega}$ with $\Omega$ contractible for which $(0.2)$ has a solution, provided $n>3$. However, for cones the condition $n>3$ is crucial for the approach in [DN] (personal communication with Professor N. Dancer). 
Theorem 4 indicates that under some topological conditions on $\Omega$ as in [BC], there should be a regular solution of $(0.2)$. In fact we can reduce our problem to that of Bahri and Coron by some simple transformations.

Theorem 5. Take $\nu=0$ and $\mathscr{C}=\mathscr{C}_{\Omega}$ and assume that $\overline{\mathscr{C}}_{\Omega} \neq \mathbf{R}^{n}$. If $H_{d}\left(\Omega, \mathbf{Z}_{2}\right)$ $\neq 0$ for some positive integer $d$, then (0.2) has a solution. Especially, in dimension 3 or 4 , there is a solution provided $\Omega \subset \Sigma^{n-1}$ is not contractible.

\section{NONEXISTENCE}

This proof will be an application of a Pohozaev type identity and there are only minor differences from the proof given in $[\mathrm{BE}]$.

Proof of Theorem 1. The proof will be based on a Pohozaev type identity as formulated in [DN, Lemma 3.7]. To se that the boundary terms vanish, we can argue exactly as in the proof of Theorem 4.1 of [BE].

If $u$ solves (0.2) we get with $K=|x|^{\nu}$ and $\tilde{x}=x-x_{0}$,

$$
\int_{\mathscr{C}}\left(\left(\frac{n}{p+1}-\frac{n-2}{2}\right) K+\frac{1}{p+1} \tilde{x} \cdot \nabla K\right) u^{p+1} d x=\frac{1}{2} \int_{\partial \mathscr{C}}(\tilde{x} \cdot \mathbf{n})|\nabla u|^{2} d \omega,
$$

where $\mathbf{n}$ is the outer normal and $d \omega$ is the surface measure. In [DN] the identity is given with $x_{0}=0$, however a simple translation gives us the formula above.

This reduces to

$$
2 \nu \int_{\mathscr{C}}\left(x_{0} \cdot x\right)|x|^{\nu-2} u^{p+1} d x+(p+1) \int_{\partial \mathscr{C}}\left(x-x_{0}\right) \cdot \mathbf{n}|\nabla u|^{2} d \omega=0 .
$$

If we take $x_{0}$ as specified in Theorem 1, then it follows that both integrals are positive and since $\nu \geq 0$ we arrive at the desired conclusion and the result follows.

In the introduction we also mentioned that in the special case when $\nu=-2$ and $p=1$, there are no regular solutions of $(0.1)$. This can be proved using some ideas from $[\mathrm{BE}]$.

Assume that $u$ satisfies $(0.1)$ with $\nu=-2$ and $p=1$. Let $\omega$ and $\psi$ be the first eigenvalue and eigenfunction of the Laplace Beltrami operator on $\Omega$, with zero boundary data. We will assume that $\psi$ is positive and has $L^{1}$ norm one (with respect to the standard surface measure). As in [BE] put $\tilde{u}(r)=$ $\int_{\Omega} u(r, \theta) \psi(\theta) d \theta$. The same argument as in [BE, §2.1] shows that $\tilde{u}$ solves

$$
\tilde{u}^{\prime \prime}+\frac{n-1}{r} \tilde{u}^{\prime}+\frac{1-\omega}{r^{2}} \tilde{u}=0 .
$$

Thus if $\alpha_{1,2}$ solves $\alpha^{2}+(n-2) \alpha+1-\omega=0$, then the solution is given by $\tilde{u}(r)=c_{1} r^{\alpha_{1}}+c_{2} r^{\alpha_{2}}$ if $\alpha_{1} \neq \alpha_{2}$ and by $\tilde{u}(r)=r^{\alpha_{1}}\left(c_{1}+c_{2} \log (r)\right)$ if $\alpha_{1}=\alpha_{2}$. Here $c_{1,2}$ are arbitrary constants.

This shows that we cannot have a regular solution. In fact it also shows that we cannot have a solution in $\mathscr{D}_{0}^{1,2}(\mathscr{C})$. This follows from the inequalities

$$
\int_{\mathscr{C}}|\nabla u|^{2} d x \geq C \int_{\mathscr{C}} u^{2}|x|^{-2} d x \geq C \int_{0}^{\infty} \tilde{u}^{2} r^{n-3} d r=\infty .
$$

Here we used both Hardy's and Jensen's inequality. 
In particular, we see that we cannot find a nontrivial function that gives equality in Hardy's inequality. If we take $p=1$ and $\nu=-2$ in the inequality in the Corollary of Theorem 2, the result is just Hardy's inequality.

\section{EXISTENCE RESULTS}

The proofs of Theorem 2 and 3 are typical applications of the ConcentrationCompactness principle from [LI]. However, since our situation is simple, we will give all the details here to make the paper self-contained.

Proof of Theorem 2. We know that the imbedding $\mathscr{D}_{0}^{1,2}(\mathscr{C}) \hookrightarrow L^{p+1}\left(\mathscr{C},|x|^{\nu} d x\right)$ is continuous if $\nu \leq 0$ (references quoted below). On the other hand a simple dilation argument shows that the imbedding is not compact.

We will consider the minimization problem

$$
S=\inf _{u \in \mathscr{D}_{0}^{1,2}(\mathscr{C})} \frac{\|\nabla u\|_{2}^{2}}{\|u\|_{p+1,|x|^{\nu}}^{2}}=\inf _{u \in \mathscr{D}_{0}^{1,2}(\mathscr{C})} \mathscr{F}(u) .
$$

The fact that $S$ is positive when $\nu<0$ can be found in [EG1] or [LI]. In fact when $\mathscr{C}=\mathbf{R}^{n}$, then $\mathscr{F}$ attains its minimum and the minimizer can be calculated explicitly and is unique modulo dilations.

Next we will consider a scaling argument. Define a continuous function $G: \mathscr{D}_{0}^{1,2}(\mathscr{C}) \rightarrow[0, \infty)$ by

$$
G(u)=S^{(p+1) / 2} \int_{\mathscr{C}} \frac{|x|}{1+|x|}|u|^{p+1}|x|^{\nu} d x .
$$

If $u \in \mathscr{D}_{0}^{1,2}(\mathscr{C})$ is nontrivial, then the scaling $\lambda^{(n-2) / 2} u(\lambda x)$ does not change the $\mathscr{D}_{0}^{1,2}(\mathscr{C})$ nor or the value of $\mathscr{F}$. On the other hand it is easy to verify that $G\left(\lambda^{(n-2) / 2} u(\lambda x)\right)$ is continuous in $\lambda$, tends to 0 , as $\lambda \rightarrow \infty$ and tends to $S^{(p+1) / 2}\|u\|_{p+1,|x|^{\nu}}^{p+1} \leq\|\nabla u\|_{2}^{p+1}$, as $\lambda \rightarrow 0$.

Let $\left\{u_{m}\right\}$ be a minimizing sequence such that $\left\|\nabla u_{m}\right\|_{2}=1$, then (after choosing a subsequence) we have $u_{m} \rightarrow u$ weakly in $\mathscr{D}_{0}^{1,2}(\mathscr{C})$, $L^{p+1}\left(\mathscr{C},|x|^{\nu} d x\right)$ and a.e. in $\mathscr{C}$. Furthermore, we can assume that the convergence is strong in $L^{p+1}\left(\left\{x \in \mathscr{C}: M^{-1}<|x|<M\right\},|x|^{\nu} d x\right)$ for any positive $M$. Finally, by the scaling properties mentioned above, we can assume that $G\left(u_{m}\right)=1 / 2$.

Before continuing we would like to clear up a technical point. When talking about measures we mean measures with finite total mass on $\mathbf{R}^{n} \cup\{\infty\}$. The space $\mathbf{R}^{n} \cup\{\infty\}$ is given the standard topology that makes it compact. This means that the measures can be identified as the dual of the space $C\left(\mathbf{R}^{n} \cup\{\infty\}\right)$. For example $\delta_{\infty}$ is well defined and $\delta_{\infty}(\varphi)=\varphi(\infty)$. In particular, the function defined by $x \rightarrow|x| /(1+|x|)$, if $x \in \mathbf{R}^{n}$ and $\infty \rightarrow 1$ is in $C\left(\mathbf{R}^{n} \cup\{\infty\}\right)$ (it is used in the definition of $G)$. Of course, we can extend $u \in \mathscr{D}_{0}^{1,2}(\mathscr{C})$ to a function in $\mathscr{D}_{0}^{1,2}\left(\mathbf{R}^{n}\right)$, by taking it to be zero outside $\mathscr{C}$. When convenient we will use this fact without warning.

Thus with the notation above we can conclude that $\left|u_{m}\right|^{p+1}|x|^{\nu} \rightarrow \gamma=$ $|u|^{p+1}|x|^{\nu}+\alpha \delta_{0}+\beta \delta_{\infty}$ as $m \rightarrow \infty$, as a measure, with $\alpha, \beta$, nonnegative (of course we might need to select a subsequence). 
Furthermore, as in [LI], we can show that

$$
\begin{aligned}
&\left|\nabla u_{m}\right|^{2} \rightarrow \mu \geq|\nabla u|^{2}+S \alpha^{2 /(p+1)} \delta_{0}+S \beta^{2 /(p+1)} \delta_{\infty}, \\
& \text { as } m \rightarrow \infty, \text { where } \alpha, \beta \geq 0 .
\end{aligned}
$$

For the sake of completeness we will show this after we have completed the proof of Theorem 2 .

It is clear that $\mu\left(\mathbf{R}^{n} \cup\{\infty\}\right)=S\left(\gamma\left(\mathbf{R}^{n} \cup\{\infty\}\right)\right)^{2 /(p+1)}$ and thus from the above we obtain

$$
\|u\|_{p+1,|x|^{\nu}}^{2}+\alpha^{2 /(p+1)}+\beta^{2 /(p+1)} \leq\left(\|u\|_{p+1,|x|^{\nu}}^{p+1}+\alpha+\beta\right)^{2 /(p+1)} .
$$

From this inequality we conclude that exactly one of $\alpha, \beta$ and $\|u\|_{p+1,|x|^{\nu}}^{p+1}$ is nonzero and thus has value $S^{-(p+1) / 2}$. Combining this with the fact that $G\left(u_{m}\right)$ tends to one if only $\beta$ is nonzero, 0 if only $\alpha$ is nonzero. We conclude that $\alpha=\beta=0$ and therefore $u_{m} \rightarrow u$ in $L^{p+1}\left(\mathscr{C},|x|^{\nu} d x\right)$. Thus by a semicontinuity argument we find that $u$ is a nontrivial minimizer of $\mathscr{F}$ and the proof of Theorem 2 is complete.

Let us prove (2.1), the proof is taken from [LI] (proof of Lemma I.1(ii)).

Put $v_{m}=u_{m}-u$, then $v_{m}$ converges weakly to zero in $\mathscr{D}_{0}^{1,2}(\mathscr{C})$ and $L^{p+1}\left(\mathscr{C},|x|^{\nu} d x\right)$ and also a.e. in $\mathbf{R}^{n}$.

Take $\varphi \in C^{1}\left(\mathbf{R}^{n} \cup\{\infty\}\right)$, such that $\nabla \varphi$ has compact support in $\mathbf{R}^{n}$. Then as $m \rightarrow \infty$ we get

$$
\int\left|\nabla v_{m}\right|^{2} \varphi^{2} d x=\int\left|\nabla\left(v_{m} \varphi\right)\right|^{2} d x+o(1) \geq S\left(\int\left|\varphi v_{m}\right|^{p+1}|x|^{\nu} d x\right)^{2 /(n-2)}+o(1) .
$$

As $m \rightarrow \infty$ we have, with convergence as measures

$$
\begin{aligned}
& \left|\nabla v_{m}\right|^{2}=\left|\nabla u_{m}\right|^{2}-2 \nabla u \cdot \nabla u_{m}+|\nabla u|^{2} \rightarrow \mu^{\prime}=\mu-|\nabla u|^{2}, \\
& \left|v_{m}\right|^{p+1}|x|^{\nu}=\left|u_{m}-u\right|^{p+1}|x|^{\nu} \rightarrow \gamma^{\prime}=\gamma-|u|^{p+1}|x|^{\nu}=\alpha \delta_{0}+\beta \delta_{\infty} .
\end{aligned}
$$

To get the last identity we used the following result by Brezis and Lieb [BrL]

$$
\int \psi\left|u_{m}-u\right|^{p+1}|x|^{\nu} d x=\int \psi\left|u_{m}\right|^{p+1}|x|^{\nu} d x-\int \psi|u|^{p+1}|x|^{\nu} d x+o(1),
$$

which holds for any $\psi \in C\left(\mathbf{R}^{n} \cup\{\infty\}\right)$.

Thus we obtain

$$
\int \varphi^{2} d \mu^{\prime} \geq S\left(\int|\varphi|^{p+1} d \gamma^{\prime}\right)^{2 /(p+1)} .
$$

Choosing different testfunctions $\varphi$, with support near 0 and $\infty$, yields $\mu^{\prime} \geq$ $S\left(\alpha^{2 /(p+1)} \delta_{0}+\beta^{2 /(p+1)} \delta_{\infty}\right)$, and the claim follows.

For the proof of Theorem 3 we need some imbedding results. Let $\mathscr{C}$ be as described in Theorem 3. Define $\mathscr{R}_{0}^{1,2}(\mathscr{C})$, to be the functions in $\mathscr{D}_{0}^{1,2}(\mathscr{C})$, that are invariant under rotations around the $z$-axis. Then we have the following imbedding result.

Lemma 6. Let $p+1=2(n+\nu) /(n-2)$, then $\mathscr{R}_{0}^{1,2}(\mathscr{C}) \hookrightarrow L^{p+1}\left(\mathscr{C},|x|^{\nu} d x\right)$ is continuous. Furthermore, any bounded sequence in $\mathscr{R}_{0}^{1,2}(\mathscr{C})$ contains a subsequence that converges in $L^{p+1}\left(\left\{x=(y, z) \in \mathscr{C}: M^{-1}<z<M\right\}\right)$, for any finite positive $M$.

The proof of Lemma 6 is based on the following elementary result. 
Lemma 7. Given $q \geq 1$, there is a constant $C$ such that for any $k \in \mathbf{Z}$

$$
\|v\|_{q}^{2} \leq C 2^{k(2 n-q(n-2)) / q}\|\nabla v\|_{2}^{2}
$$

holds for all $v \in \mathscr{R}_{0}^{1,2}(\mathscr{C})$, with $\operatorname{supp} v \subset\left\{(y, z) \in \mathscr{C}: 2^{k-1}<z<2^{k+2}\right\}$.

Proof of Lemma 7. It is enough to prove the inequality for $k=0$ the rest follows by a dilation.

By the symmetry, $v=v(|y|, z)$ is a function of two variables, $d x=$ $\omega_{n-2}|y|^{n-2} d|y| d z$, where $\omega_{n-2}$ is the volume of the $n-2$ sphere. The support of $v$ is contained in the domain $\left\{(y, z): \frac{1}{2}<z, d z<|y|<D z\right\}$, which is bounded away from the origin. Hence the result follows from the Sobolev imbedding theorem in dimension 2.

By the same argument as above it also follows that the imbedding $\mathscr{R}_{0}^{1,2}(\mathscr{C}) \cap$ $\left\{u: \operatorname{supp} u \subset\left\{(y, z) \in \mathscr{C}: M^{-1}<z<M\right\}\right\} \hookrightarrow L^{q}$ is compact for any $q>1$ and $M<\infty$.

Proof of Lemma 6. The existence of a subsequence convergent away from the origin and infinity follows from the last remark in the proof of Lemma 7 and a diagonalization procedure.

Next we prove that the imbedding is continuous. Take

$$
\varphi(s)= \begin{cases}s-1 & \text { if } 1 \leq s \leq 2 \\ 1 & \text { if } 2 \leq s \leq 4 \\ \frac{8-s}{4} & \text { if } 4 \leq s \leq 8 \\ 0 & \text { otherwise }\end{cases}
$$

and take $\eta_{k}=\varphi\left(2^{-k} s\right)^{2}$. Furthermore, let $\chi_{k}(s)$ be one if $2^{k} \leq s \leq 2^{k+3}$ and zero otherwise. Then $\chi_{k}$ and $\eta_{k}$ have the same support and $\left|\left(\sqrt{\eta_{k}(s)}\right)^{\prime}\right|=$ $\left|2^{-k} \varphi^{\prime}\left(2^{-k} s\right)\right| \leq(1 / s) \chi_{k}(s)$. Also it is easy to verify that $1 \leq \sum_{k=-\infty}^{\infty} \eta_{k}(s) \leq 3$ and $1 \leq \sum_{k=-\infty}^{\infty} \chi_{k}(s) \leq 3$, for $s>0$.

Note that if $x=(y, z) \in \mathscr{C}$, then $d^{\prime}|x| \leq z \leq D^{\prime}|x|$ for some positive numbers $d^{\prime}$ and $D^{\prime}$. Now if we take $\eta_{k}$ to be a function of $z$ only (i.e. $\eta_{k}=\eta_{k}(z)$, where $\left.x=(y, z)\right)$, we obtain

$$
\begin{aligned}
& \|u\|_{p+1,|x|^{\nu}}^{2} \leq\left\|\sum_{k=-\infty}^{\infty} \eta_{k} u^{2}\right\|_{(p+1) / 2,|x|^{\nu}} \\
& \quad \leq \sum_{k=-\infty}^{\infty}\left\|\eta_{k} u^{2}\right\|_{(p+1) / 2,|x|^{\nu}} \leq\left(d^{\prime}\right)^{-2 \nu /(p+1)} 2^{6 \nu /(p+1)} \sum_{k=-\infty}^{\infty} 2^{k 2 \nu /(p+1)}\left\|\sqrt{\eta_{k}} u\right\|_{p+1}^{2} \\
& \quad \leq C \sum_{k=-\infty}^{\infty} 2^{k(2 \nu+2 n-(p+1)(n-2)) /(p+1)}\left\|\nabla\left(\sqrt{\eta_{k}} u\right)\right\|_{2}^{2} \\
& \quad \leq C \sum_{k=-\infty}^{\infty}\left(\int \eta_{k}|\nabla u|^{2} d x+\int u^{2}|x|^{-2} \chi_{k} d x\right) \leq C\|\nabla u\|_{2}^{2} .
\end{aligned}
$$

In the last step we used the Hardy inequality $\|u\|_{2,|x|^{-2}} \leq C\|\nabla u\|_{2}$.

Proof of Theorem 3. The proof is almost the same as that of Theorem 2. We minimize the functional $\mathscr{F}$ over $\mathscr{R}_{0}^{1,2}(\mathscr{C})$. By Lemma $6, \mathscr{F}$ is bounded from 
below by a positive constant. Using Lemma 6 again, we see that the only way a minimizing sequence $\left\{u_{m}\right\}$ can fail to converge in $L^{p+1}\left(\mathscr{C},|x|^{\nu} d x\right)$, is that $\left|u_{m}\right|^{p+1}|x|^{\nu}$ concentrates at the origin or at infinity. However, by scaling as in the proof of Theorem 2, we can avoid this.

Next we will prove Theorem 4 . The argument is almost the same as that in $[\mathrm{CO}]$ and [DI].

Proof of Theorem 4. Define $\mathcal{J}: M \rightarrow \mathbf{R}$ where

$$
\begin{aligned}
& \mathscr{J}(u)=\frac{1}{\|u\|_{p+1}^{n}}, \\
& M=\left\{u \in \mathscr{D}_{0}^{1,2}(\mathscr{C}):\|\nabla u\|_{2}=1, u \not \equiv 0\right\} \text { and } M_{+}=\{u \in M: u \geq 0\}
\end{aligned}
$$

Then with the previous definition of $S$, we have $S^{n / 2}=\inf _{u \in \mathscr{D}_{0}^{1,2}(\mathscr{C})} \mathcal{J}(u)$. Furthermore, identifying the dual of $\mathscr{D}_{0}^{1,2}(\mathscr{C})$ with $\mathscr{D}_{0}^{1,2}(\mathscr{C})$ itself as usual, the differential of $\mathscr{J}$ at $v \in M_{+}$is given by

$$
\nabla \mathscr{J}(v)=n \mathscr{J}(v)\left(v-\mathscr{J}(v)^{2 /(n-2)}(-\Delta)^{-1}\left(|v|^{4 /(n-2)} v\right)\right) .
$$

Thus if $u$ is a critical point of $\mathscr{J}$ and $u$ is a nonnegative, then $\sqrt{\mathscr{J}(u)} u$ solves $(0.2)$.

Define $f: M_{+} \rightarrow \mathbf{R}_{+}^{n}, g: \mathbf{R}_{+}^{n} \rightarrow H^{n-1}=\left\{(y, z) \in \mathbf{R}^{n-1} \times \mathbf{R}: z=1\right\}$ and $F=g \circ f: M_{+} \rightarrow H^{n-1}$, by

$$
f(u)=\int \frac{x}{1+|x|}|\nabla u|^{2} d x \text { and } g(y, z)=\left(z^{-1} y, 1\right) .
$$

Note that these mappings are continuous.

Take $\psi, \varphi_{k} \in C^{\infty}(\mathbf{R},[0,1])$ for $k \in \mathbf{Z}_{+}$, such that $\psi(s)$ is 1 if $|s-1|<$ $1 / 2$, is 0 if $|s-1|>3 / 4$ and such that $\varphi_{k}$ is 0 outside $(1 / 4 k, 4 k)$, is one in $[1 / 2 k, 2 k]$ and such that $\left|\nabla \varphi_{k}\right|$ is less than $3 k$ in $[1 / 4 k, 1 / 2 k]$ and less than $1 / k$ in $[2 k, 4 k]$.

Put

$u_{t}^{\sigma}(x)=\left(\frac{\mu(1-t)}{\mu^{2}(1-t)^{2}+|x-t \bar{\sigma}|^{2}}\right)^{(n-2) / 2} \quad$ with $t \in[0,1), \bar{\sigma}=(\sigma, 1) \in H^{n-1}$, where $\sigma \in \Sigma^{n-2}$ (the $n-2$ sphere).

We choose $\mu$ small positive so that $\mathscr{J}\left(P\left(\psi u_{t}^{\sigma}\right)\right) \leq(11 / 10) S^{n / 2}$, for $t \in$ $[0,1)$ and $\sigma \in \Sigma^{n-2}$, where $\psi=\psi(z)$ and $P(w)=|w| /\|\nabla w\|_{2}$.

Finally put

$$
v_{k, t}^{\sigma}(x)=v_{k, t}^{\sigma}(y, z)=P\left(\varphi_{k}\left(\frac{|y|}{z}\right) \psi(z) u_{t}^{\sigma}(y, z)\right) .
$$

We have the following lemma (the same as Lemma 2 in [CO]).

Lemma 8. (i) For all $\varepsilon>0$, there is an $\eta<1$ such that

$$
\mathscr{J}\left(v_{k, t}^{\sigma}\right) \leq S^{n / 2}+\varepsilon, \quad\left|F\left(v_{k, t}^{\sigma}\right)-\bar{\sigma}\right|<\varepsilon,
$$

for all $t \in[\eta, 1), \sigma \in \Sigma^{n-2}$ and all $k \in \mathbf{Z}_{+}$.

(ii) There is an integer $k_{0}$ such that $\mathscr{J}\left(v_{k, t}^{\sigma}\right) \leq \frac{3}{2} S^{n / 2}$, for all $t \in[0,1)$, $k \geq k_{0}$ and all $\sigma \in \Sigma^{n-2}$.

The proof is rather straightforward and is omitted.

The next lemma concerns the level sets $J_{+}^{b}=\left\{u \in M_{+}: \mathscr{J}(u) \leq b\right\}$. 
Lemma 9. Given any open set $G \supset \overline{\mathscr{C}}$, there is an $\varepsilon>0$, such that $F\left(J_{+}^{S^{n / 2}+\varepsilon}\right) \subset$ $G \cap H^{n-1}$.

The proof of this lemma requires some additional analysis to the argument given in [CO]. The reason is that $\mathscr{C}$ is unbounded. We will give the argument after finishing the proof of Theorem 4.

Define a mapping $\gamma:[0,1) \rightarrow C^{0}\left(\Sigma^{n-2}, M_{+}\right)$by $\gamma(t): \sigma \rightarrow v_{k_{0}, t}^{\sigma}$, where $k_{0}$ is as in Lemma 8(ii). This means that the support of $v_{k_{0}, t}^{\sigma}$ is contained in $\mathscr{C}$, provided we pick $\alpha=4 k_{0}$.

Collecting the results above we get:

(I) By Lemma 8(i) and Lemma 9, there are $0<\varepsilon$ and $t_{0}<1$ such that $\gamma\left(t_{0}\right) \in J_{+}^{S^{n / 2}+\varepsilon}$ is not homotopic to any constant mapping in $C^{0}\left(\Sigma^{n-2}, J_{+}^{S^{n / 2}+2 \varepsilon}\right)$.

(II) By Lemma 8 (ii), $\gamma\left(t_{0}\right)$ is homotopic to the constant mapping $\gamma(0)$ in $C^{0}\left(\Sigma^{n-2}, J_{+}^{\left(\frac{3}{2} S^{n / 2}\right.}\right)$.

Now using the fact that $M_{+}$is invariant under the flow generated by $-\nabla \mathscr{J}$, it follows that $\nabla \mathscr{J}$ cannot be bounded away from 0 on the set $J_{+}^{\frac{3}{2}} S^{n / 2} \backslash J_{+}^{S^{n / 2}+2 \varepsilon}$. Thus, there is a sequence $\left\{u_{m}\right\}$ such that $\mathscr{J}\left(u_{m}\right) \rightarrow c \in\left[S^{n / 2}+2 \varepsilon, \frac{3}{2} S^{n / 2}\right]$ and $\nabla \mathscr{J}\left(u_{m}\right) \rightarrow 0$, as $m \rightarrow \infty$.

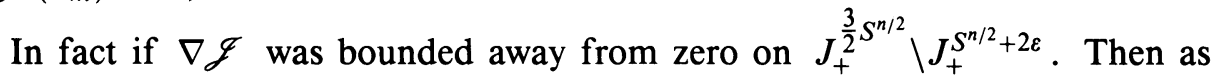
in [RA, Appendix A], we can construct a modified negative gradient flow $\eta$ : $\mathbf{R} \times M_{+} \rightarrow M_{+}$such that

$$
\begin{aligned}
& \eta(0, \cdot)=\text { identity mapping, } \\
& \eta\left(1, J_{+}^{\frac{3}{2} S^{n / 2}}\right) \subset J_{+}^{S^{n / 2}+2 \varepsilon}, \\
& \eta\left(s, \gamma\left(t_{0}\right)\right)=\gamma\left(t_{0}\right), \quad \text { for any } s \in[0,1] .
\end{aligned}
$$

However, this is not consistent with (I) and (II) above, giving us the desired contradiction.

Thus the first part in Theorem 4 follows from the following result.

Lemma 10. The functional $\mathscr{J}$ satisfies $(P S)_{c}$, for any $c \in\left(S^{n / 2}, 2 S^{n / 2}\right)$.

The proof is essentially contained in Struwe [ST]. In Struwe it is assumed that the domain is bounded and the functional is slightly different. However, the extensions to unbounded domains and the functional $\mathscr{F}$ are straightforward.

Next we will make the necessary changes in order to obtain part two.

Take $\eta \in C^{\infty}(\mathbf{R},[0,1])$ to be a nonincreasing function such that $\eta(s)=1$ if $s<1 / 2, \eta(s)=0$ if $s>1$. For $\delta$ positive, define $\eta_{\delta}(x)=\eta_{\delta}\left(y_{1}, y^{\prime}, z\right)=$ $\eta\left(2 \delta y_{1} /\left(\left|y^{\prime}\right|+\delta y_{1}\right)\right.$, and $v_{k_{0}, t, \delta}^{\sigma}=P\left(\eta_{\delta} v_{k_{0}, t}^{\sigma}\right) \in \mathscr{D}_{0}^{1,2}(\mathscr{C})$.

If $n>4$, then it follows that $v_{k_{0}, t, \delta}^{\sigma} \rightarrow v_{k_{0}, t}^{\sigma}$ in $\mathscr{D}_{0}^{1,2}(\mathscr{C})$, as $\delta \rightarrow 0$. Thus choosing $\delta$ small enough we can argue as in part one above to obtain a solution.

The assumption that $n>4$ was used to conclude that $\left|\nabla \eta_{\delta}\right|^{2} \rightarrow 0$ in $L^{1}$ on the support of $v_{k_{0}, t}^{\sigma}$, as $\delta \rightarrow 0$.

Proof of Lemma 9. Let us assume that there is a sequence $\left\{u_{m}\right\}$ such that $u_{m} \in \mathscr{J}_{+} S^{n / 2+1 / n}$ and $d\left(F\left(u_{m}\right), H^{n-1} \cap \mathscr{C}\right)>\delta>0$ uniformly in $n$. Then, since 
$\mathscr{F}$ does not attain its minimum, it follows from [LI], that for a subsequence we get $\left|\nabla u_{m}\right|^{2} \rightarrow \delta_{x_{0}}$, where $x_{0} \in \overline{\mathscr{C}} \cup\{\infty\}$.

If $x_{0} \neq 0$ and $x_{0} \neq \infty$, then $g\left(x_{0}\right) \in H^{n-1} \cap \overline{\mathscr{C}}$, and we obtain a contradiction.

If $x_{0}=\infty$ we argue as follows. Put $v_{m}(x)=\lambda_{m}^{(n-2) / 2} u_{m}\left(\lambda_{m} x\right)$, where $\lambda_{m}$ is chosen so that $\int(|x| /(1+|x|))\left|\nabla v_{m}(x)\right|^{2} d x=\frac{1}{2}$. Note that $\lambda_{m} \rightarrow \infty$, as $m \rightarrow \infty$.

Then, since $v_{m}$ is also a minimizing sequence for $\mathscr{J}$, we have (choosing a subsequence again) $\left|\nabla v_{m}\right|^{2} \rightarrow \delta_{x_{0}^{\prime}}$, where $x_{0}^{\prime} \in \overline{\mathscr{C}}$ and $x_{0}^{\prime}$ is not zero or infinity. Thus going back we get

$$
f\left(u_{m}\right)=\int \frac{x}{|1+| x \mid}\left|\nabla u_{m}\right|^{2} d x=\int \frac{\lambda_{m} x}{1+\lambda_{m}|x|}\left|\nabla v_{m}\right|^{2} d x \rightarrow \frac{x_{0}^{\prime}}{\left|x_{0}^{\prime}\right|} \in \overline{\mathscr{C}},
$$

as $m \rightarrow \infty$. Therefore we conclude that $\lim _{m \rightarrow \infty} F\left(u_{m}\right) \in H^{n-1} \cap \overline{\mathscr{C}}$, and we get a contradiction again.

The argument when $x_{0}=0$ is very much the same. If we rescale as above we find that $\lambda_{m} \rightarrow 0$, as $m \rightarrow \infty$. Therefore, with $\left|\nabla v_{m}\right|^{2} \rightarrow \delta_{x_{0}^{\prime}}$ we find that $\lambda_{m}^{-1} f\left(u_{m}\right) \rightarrow x_{0}^{\prime}$ and thus $F\left(u_{m}\right) \rightarrow g\left(x_{0}^{\prime}\right) \in H^{n-1} \cap \overline{\mathscr{C}}$. This completes the proof.

For an unbounded domain $D \subset \mathbf{R}^{n}$, consider the problem of finding positive solutions of

$$
\Delta u+u^{(n+2) /(n-2)}=0, \quad \text { in } D, \quad u \in \mathscr{D}_{0}^{1,2}(D) .
$$

A solution of (2.2) is smooth and has the asymptotic decay $u(x)=O\left(|x|^{2-n}\right)$, as $D \ni x \rightarrow \infty$.

If $D$ is the complement of a starshaped domain, a direct application of the Pohozaev identity shows that (2.2) does not have a solution.

The reason why the argument of Coron, with $D$ the complement of a small hole, does not work here is that Lemma 9 is no longer true. In fact this can be understood as follows. The point $\infty$ is not different from any other point in $\mathbf{R}^{n}$, for this equation. If we consider $D$ as a subset of the compactified $\mathbf{R}^{n} \cup\{\infty\}$, which is more natural. Then $D$ is contractible and $D$ is starshaped with respect to $\infty$.

Let us transform (2.2) as follows. We will assume that the interior of the complement of $D$ contains the origin (note that the problem is translation invariant). Let $\widetilde{D}$ be the image of $D$ under inversion $x \rightarrow x /|x|^{2}$, of course this mapping is a homeomorphism on $\mathbf{R}^{n} \cup\{\infty\}$. If the function $u$ in (2.2) is transformed using the Kelvin transform $u \rightarrow K(u)=|x|^{2-n} u\left(x /|x|^{2}\right)$. Then $K(u)$ solves $(2.2)$ with $D$ replaced by the bounded domain $\widetilde{D}$. Note that the Kelvin transform is an isometry on $\mathscr{D}_{0}^{1,2}\left(\mathbf{R}^{n}\right)$. For a proof that the Kelvin transform is continuous see for example [EG2] (Lemma 8).

Now we can apply the result of Bahri and Coron [BC] to this problem and transform it back. The sets $D$ in $\mathbf{R}^{n} \cup\{\infty\}$ and $\widetilde{D}$ in $\mathbf{R}^{n}$ are homeomorphic and thus generate the same homology. Thus we have obtained the following variant of Bahri's and Coron's result.

Theorem 11. If the complement of $D \subset \mathbf{R}^{n} \cup\{\infty\}$ has nonempty interior and $H_{d}\left(D, Z_{2}\right)$ is nontrivial, for some positive integer $d$, then (2.2) has a solution. 
In particular, if $n=3$ and $D$ is not contractible in $\mathbf{R}^{3} \cup\{\infty\}$, then (2.2) has a solution.

To prove Theorem 5 , we note that $H_{d}\left(\mathscr{C}_{\Omega}, \mathbf{Z}_{2}\right)=H_{d}\left(\Omega, \mathbf{Z}_{2}\right),\left(\mathscr{C}_{\Omega} \subset \mathbf{R}^{n} \cup\right.$ $\{\infty\})$. From homology theory it follows that if $n=3$ or $n=4$ and $\Omega \subset \Sigma^{n-1}$ is not contractible to a point, then $H_{d}\left(\Omega, \mathbf{Z}_{2}\right)$ is nontrivial for some integer $d$.

\section{ACKNOWLEDGEMENT}

The author wishes to thank Matts Essén, for suggesting this problem and for several helpful discussions.

\section{REFERENCES}

[BC] A. Bahri and J.-M. Coron, On a nonlinear elliptic equation involving the critical Sobolev exponent: The effect of the topology of the domain, Comm. Pure Appl. Math. 41 (1988), 253-294.

[BE] C. Bandel and M. Essén, On the positive solutions of Emden equations in cone-like domains, Arch. Rational Mech. Anal. 112 (1990), 319-338.

[BaL] C. Bandle and $\mathrm{H}$. A. Levine, On the existence and nonexistence of global solutions of reactiondiffusion equations in sectorial domains, Trans. Amer. Math. Soc. 316 (1989), 595-622.

[BrL] $\mathrm{H}$. Brezis and $\mathrm{E}$. Lieb, A relation between pointwise convergence of functions and convergence of functionals, Proc. Amer. Math. Soc. 88 (1983), 486-490.

[CO] J.-M. Coron, Topologie et cas limite des injections de Sobolev, C. R. Acad. Sci. Paris 299 (1984), 209-212.

[E] E. N. Dancer, $A$ note on an equation with critical exponent, Bull. London Math. Soc. 20 (1988), 600-602.

[DI] W.-Y. Ding, Positive solutions of $\Delta u+u^{(n+2) /(n-2)}=0$ on contractible domains, J. Partial Differential Equations 2 (1989).

[DN] W.-Y. Ding and W.-M. Ni, On the elliptic equation $\Delta u+K u^{(n+2) /(n-2)}=0$ and related topics, Duke Math. J. 52 (1985), 485-486.

[EG1] H. Egnell, Elliptic boundary value problems with singular coefficients and critical nonlinearities, Indiana Univ. Math. J. 38 (1989), 235-251.

[EG2] _ Asymptotic results for finite energy solutions of semilinear elliptic equations, J. Differential Equations (to appear).

[GT] D. Gilbarg and N. S. Trudinger, Elliptic partial differential equations of second order, 2nd ed., Springer-Verlag, New York, 1983.

[LI] P.-L. Lions, The concentration-compactness principle in the calculus of variations. I, II, Riv. Mat. Iberoamericana 1 (1985), 145-201, 45-121.

[KW] J. Kazdan and F. Warner, Remarks on some quasilinear elliptic equations, Comm. Pure Appl. Math. 28 (1975), 567-597.

[PA] P. Passaseo, Multiplicity of positive solutions of nonlinear elliptic equations with critical Sobolev exponent in some contractible domains, Manuscripta Math. 65 (1989), 147-165.

[RA] P. H. Rabinowitz, Minimax methods in critical point theory with applications to differential equations, CBMS-NSF Regional Conf. Ser. in Math., no. 65, Amer. Math. Soc., Providence, R. I., 1986.

[ST] M. Struwe, A global compactness result for elliptic boundary problems involving limiting nonlinearities, Math. Z. 187 (1984), 511-517.

School of Mathematics, University of Minnesota, Minneapolis, Minnesota 55455

Current address: Department of Mathematics, Uppsala University, Thunbergsv. 3, 75238 Uppsala, Sweden 